\title{
Automobile wheel clearance estimation using magnetism
}

\author{
A. Le Goff ${ }^{a, *}$, J.-L. Lacoume ${ }^{a, b}$, R. Blanpain ${ }^{a}$, S. Dauvé ${ }^{a}$, C. Serviere ${ }^{b}$ \\ a CEA/LETI, Minatec, Grenoble, France \\ ${ }^{\mathrm{b}}$ GIPSA-lab, Groupe INP, Grenoble, France
}

\begin{abstract}
With development and miniaturization of magnetic sensors for several years, it is now possible to imagine new applications using magnetic measures for monitoring and diagnostics. In this article this new way of research and development is presented with a concrete example concerning monitoring of wheels in an automobile.

Our approach consists in using the low magnetic field created by the metallic elements of the wheel in order to localize them with low-cost high-sensitivity miniature magnetic sensors. The measures are made via a sensor network set up on a vehicle around the wheel. Then we use a physical model of the wheel in order to interpret the signals we get from the sensors. This method shows the interest of the magnetic measurement for monitoring in automobile.

In this paper we present the magnetic signal created by a wheel, the mechanical model of the wheel and we present how we use them for an application: the real-time estimation of the distance between wheels and chassis (wheel clearance).
\end{abstract}

\section{Introduction and state of the art}

During the recent years, many studies have shown the interest of vibration or acoustic analysis for automobile diagnosis. Those studies have now reached a level of maturity and it could be opportune to think about other sources of information for monitoring or diagnosis. Magnetism is already known for its abilities to detect and localize ferromagnetic objects (such as steel and iron parts) in space. It could be a good candidate.

In the past, magnetic field has been used for localizing submarines by exploiting their magnetization [1]. Thanks to the development and miniaturization of magnetic sensors, new applications appeared using a magnet attached on the object we want to localize, and a magnetic sensor [2]. Those techniques are widely used in today's products for measuring positions or rotation speeds by the use of a magnet and a Hall effect sensor.

Recently, it has been shown that it is possible to measure the rotational speed of an automobile wheel by the use of a single low-cost MR (Magneto Resistance) sensor on chip without any magnet thanks to the magnetization of the wheel [3].

In this paper we present this kind of magnetization and we use it for estimating the wheel clearance.

\section{Magnetization of automobile wheels}

As it has been shown in [3] there is a non-homogeneous magnetization in automobile wheels. In physics, magnetization $\vec{M}$ is a field inside a material measured in $A / m$. The magnetization can be seen as a source of magnetic field outside the

\footnotetext{
* Corresponding author.

E-mail address: alexis.le_goff@centraliens.net (A. Le Goff).
} 
material. One of the consequences of this magnetization is that the wheel generates a magnetic "signature" when it is rotating. We can put an AMR (anisotropic magneto resistance) magnetic sensor above the wheel in order to measure this signature. This kind of sensor only requires an instrumentation amplifier in order to have a sufficient signal and a $1000 \mathrm{~Hz}$ anti-aliasing filter. Fig. 1 shows the raw outputs of an AMR sensor located $10 \mathrm{~cm}$ above the rotating wheel. The wheel is rotating at about 2 turns per second, which corresponds to a vehicle speed of $15 \mathrm{~km} / \mathrm{h}$. The sample rate is $10 \mathrm{kHz}$. One can clearly notice on this figure a $\pm 40 \mu \mathrm{T}$ signature. By way of comparison the strength of the earth magnetic field is about $40 \mu \mathrm{T}$ in Europe.

In [4] this magnetization is modelled as a sum of magnetic dipoles. A magnetic dipole is a mathematic tool that corresponds to an area in the material where the magnetization is homogeneous. The principle of this model is to find the number and the parameters of the dipoles that minimize the distance between the measured signature and the calculated one with magnetic dipoles. This approach uses an optimization. The results from this optimization are shown in Fig. 2. In this figure the calculated signature uses five magnetic dipoles.

Fig. 2 shows that the magnetization of the wheel is located mainly in the steel belt (in dotted line) of the tire. The steel belt (or metallic carcass) is a part of the tire made of steel wires that protects the tire against centrifugal force, and punctures.

It is interesting to notice that each tire has a different magnetic signature, even for the tires of a same vehicle. This magnetization depends on several parameters like the history of the steel. The most important source of magnetization in
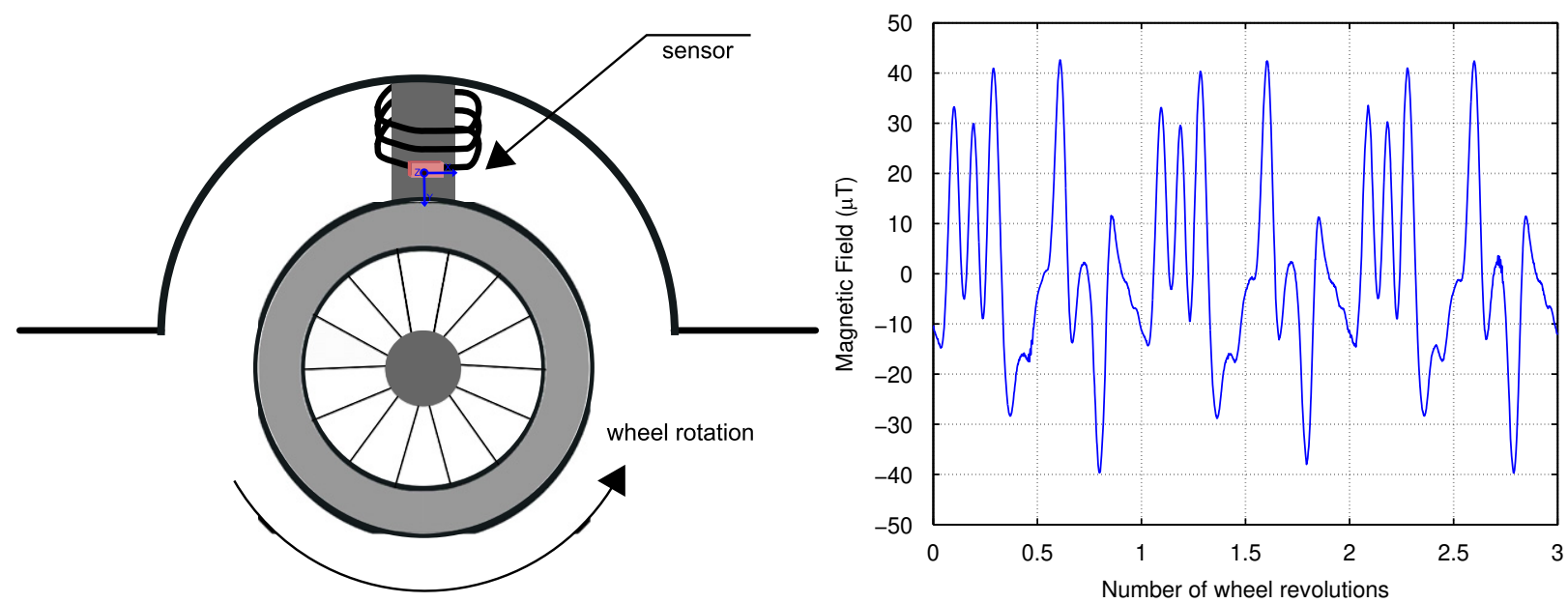

Fig. 1. Left: scheme of the measure. Right: raw output signal of the sensor in the horizontal direction.
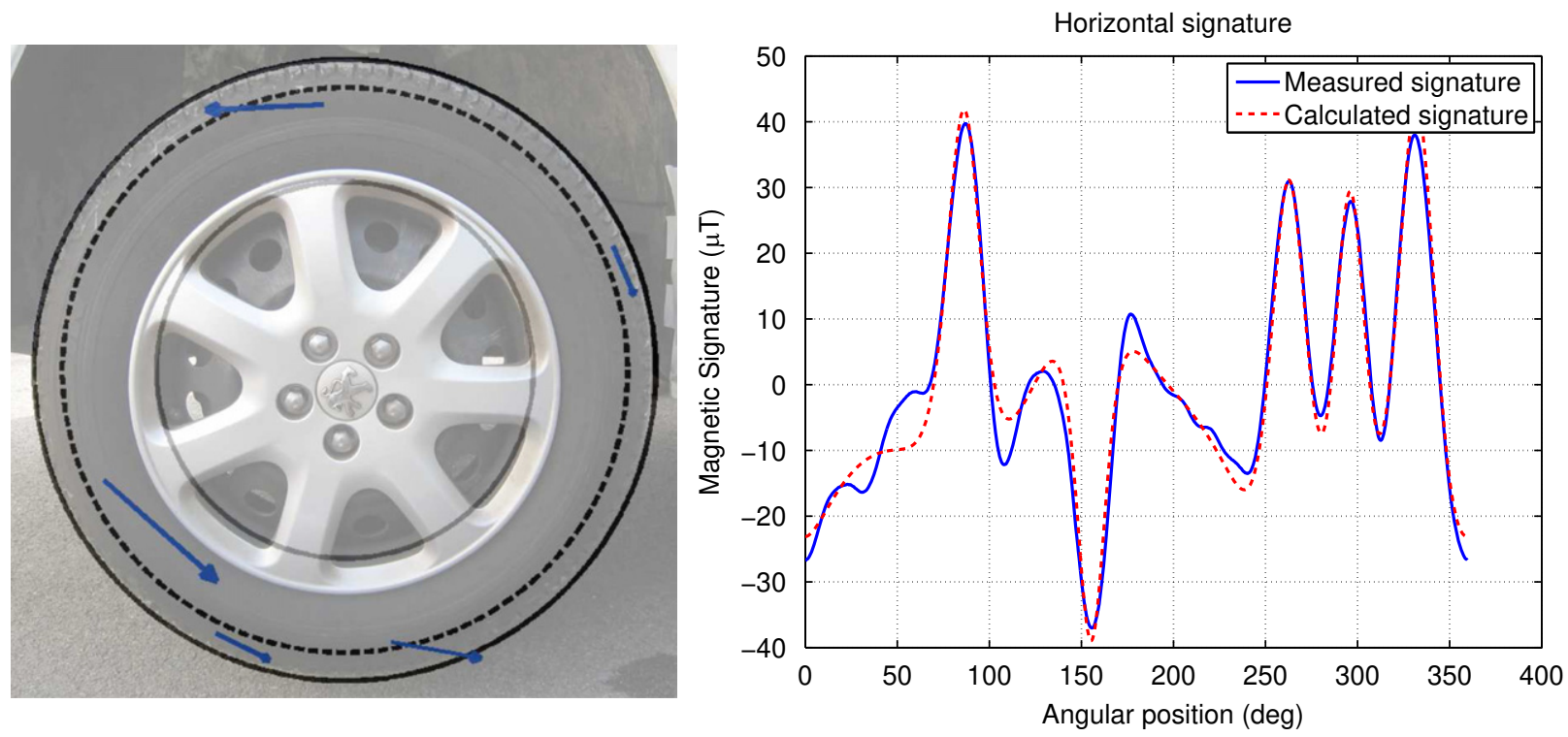

Fig. 2. Results of the optimization. Left: magnetic dipoles found after optimization. Right: measured and calculated signatures. 
the tires is the fact that they are handled with huge electromagnets in factories. Those electromagnets generate a magnetization in the steel belt of the tires.

We showed that this magnetization remains the same after one month of normal use of the vehicle. It would be interesting to study how the wheel magnetization changes in a longer time and in specific conditions.

\section{Application for wheel clearance estimation}

In automobile, the wheel clearance is by definition, the relative distance between the center of a wheel and the chassis. Wheel clearance corresponds to the suspension length. This length is a function of time because of the irregularities of the road and the inertial forces (rolling, pitching, etc.) [5].

Measuring wheel clearance in real time allows estimating the resonance frequency of the suspension by the use of the clearance spectra. Clearance of all the wheels in a vehicle allows to estimate the rolling by calculating the difference between left and right wheel clearances and the pitching with the difference between front and rear wheels.

At present there are several methods for estimating wheel clearance. One of these methods consists in using a sensor and a magnet so that the distance between the magnet and the sensor is a function of the clearance [6]. The main problem of this method is that it is necessary to fit two elements of the car (chassis and wheel) out. The application presented in this article uses two sensors located on the chassis that measure the magnetic signature from the rotating wheel.

Let us consider two magnetic sensors denoted $S_{1}$ and $S_{2}$ located on the chassis, one in front of the wheel and the other behind (Fig. 3). Since the sensors are located at the same distance from the wheel center they should deliver the same signal with the same pattern but with a temporal delay. This delay $\tau$ is given by this equation:

$$
\tau=\frac{\Psi}{2 \pi f} \quad \text { where } \Psi \text { is the angle between } \overrightarrow{G S_{1}} \text { and } \overrightarrow{G S_{2}}
$$

where $G$ is the wheel center and $f$ is the instantaneous rotation frequency of the wheel.

We consider the mudguard as a half circle of center $O$ and radius $d . S_{1}$ and $S_{2}$ are located on it, with angles $\alpha_{1}$ and $\alpha_{2}$. There is a geometrical relationship between the angle $\Psi$ and the clearance $h$ (Fig. 3). We give this relation for the simplest case (but it works also in the other cases) where we put the sensors such as $\alpha_{1}=0$ and $\alpha_{2}=\pi$ :

$$
\cos (\Psi)=\frac{h^{2}-d^{2}}{h^{2}+d^{2}}
$$

$\Psi$ corresponds to the angular delay between the two signals. It is equal to the phase difference between the two signals. It means that we can estimate the wheel clearance if we know the phase difference by reversing Eq. (2). The phase estimation can be performed with the magnetic signals by using angular resampling techniques [7].

\section{Phase estimation}

As we saw in the previous section it is necessary to estimate the phase difference between the two signals. It means that we have to estimate the phase of the both temporal signals (Fig. 4).

There are several techniques for estimating the phase of a signal with frequency fluctuations. Most of them suppose that the signal has small frequency fluctuations ( $\pm 10 \%$ ). In our case, the wheel has a wide range of rotation speeds (from 0 to $20 \mathrm{~Hz}$ ). It is therefore impossible to use such techniques. The method we use is described below.

The first step of this method is to detect the negative extrema (of course it also works with the positive ones) of the signature in the signal. Each extremum appears once per wheel revolution. We know that the instants of each peak correspond to a same angular position of the wheel (modulo $2 \pi$ ). We ascribe the phase origin to the first peak. With this arbitrary rule it means that the $n$th peak corresponds to a phase equal to $(n-1) \times 2 \pi$ (Fig. 5 ).

We now have a phase estimation for every instants corresponding to a peak but we want to have the phase for every instant between two peaks. In order to estimate the phase for every point in the signal we make a splines interpolation [8] for every point between two peaks. This interpolation amounts to making the approximation that the rotation speed of the wheel was constant between the two peaks.

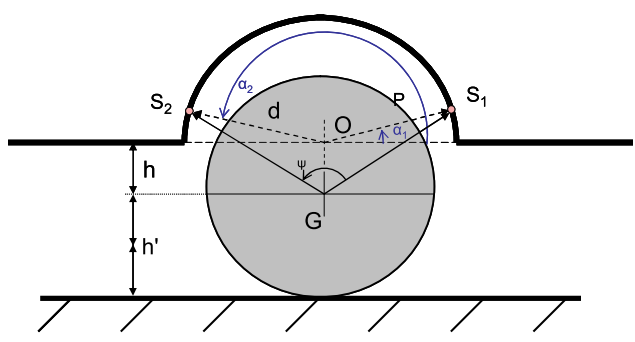

Fig. 3. $h$ is the wheel clearance. 


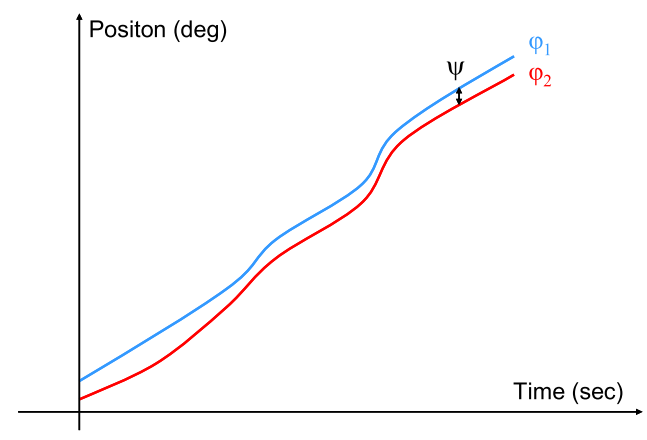

Fig. 4. Phases and phase difference.

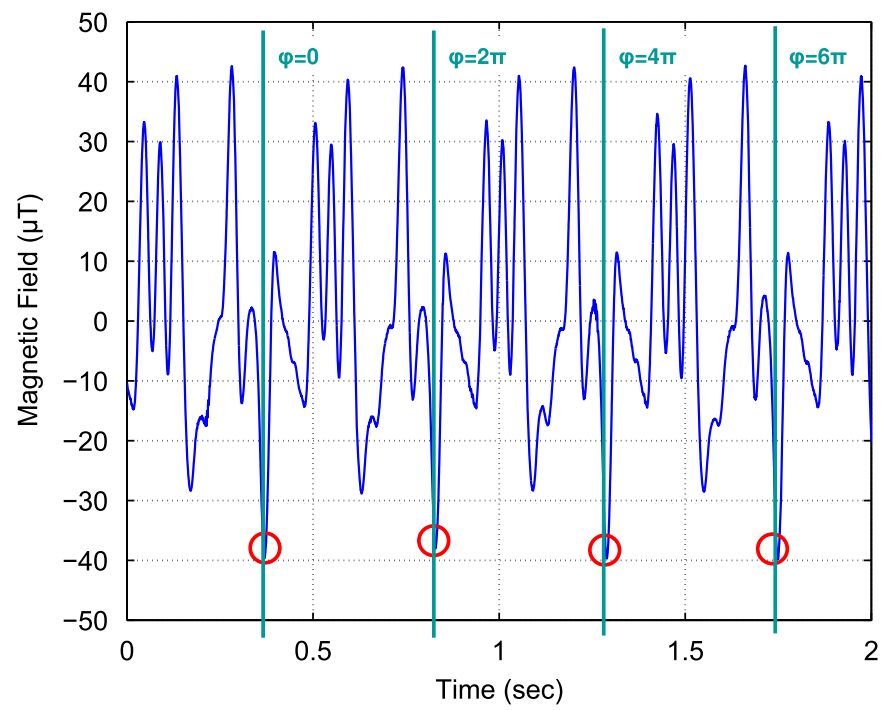

Fig. 5. Peak detection and phase estimation

With the phase estimation of each signal, we have the phase difference and then also the wheel clearance by reversing Eq. (2). The precision of this phase estimation depends on the precision of the peak detection which is dependent on the sampling rate. If we want to have a $1^{\circ}$ precision on the phase estimation when the wheel is rotating at high speed $(20 \mathrm{~Hz})$, we need to have a $20 \mathrm{~Hz} \cdot 360=7200 \mathrm{~Hz}$ sampling rate. A $1^{\circ}$ precision on the phase estimation corresponds approximately to a $2 \mathrm{~mm}$ on wheel clearance precision according to equation (2).

\section{Results and discussion}

We compared this technique with another one based on a laser sensor [9]. The laser sensor is located in front of the vehicle and it measures the exact distance between the road and the chassis. The laser sensor is used as a reference sensor. On one wheel, we put 2 magnetic sensors as in the same configuration as Fig. 3. The results of this comparison are given in Fig. 6.

As we can see in this figure, both sensors give approximately the same clearance at the beginning of the experiment when the vehicle accelerates and at the end when it decelerates. They also give similar curves for most of the experiment. As we can see, there are small differences at about 10 or $20 \mathrm{~s}$. One of the possible explanations is that the laser sensor is not a perfect reference for two reasons:

(a) the laser sensor measures the displacement of the chassis which is a combination of the four wheel clearances and

(b) the laser method measures all the irregularities of the road and the magnetic method does not since they are mechanically filtered by the tire.

In the future, it would be necessary to use a clearance sensor dedicated to the wheel in order to make a better comparison. Nevertheless this experiment demonstrates the possibilities given by the magnetism for wheel clearance estimation. 


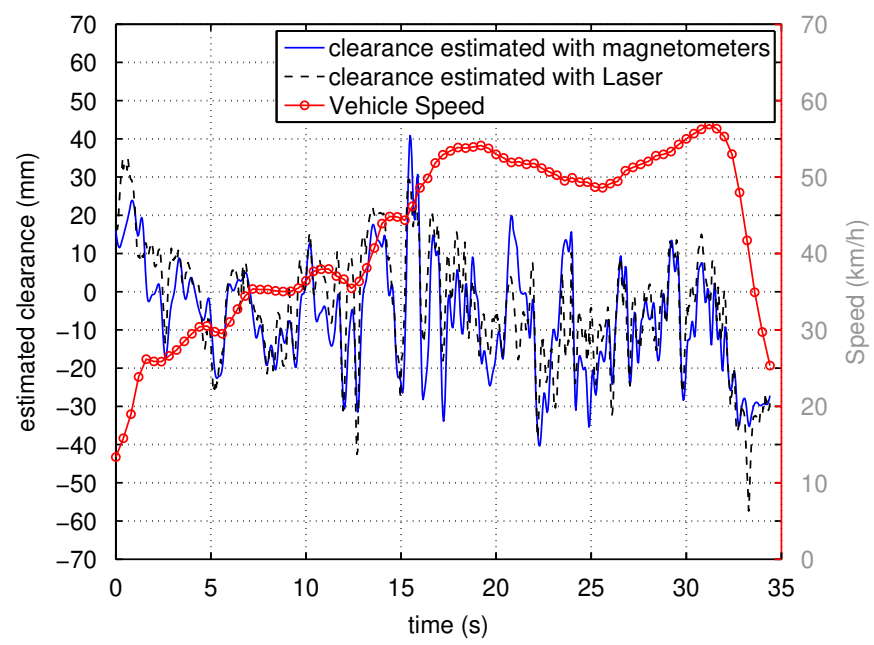

Fig. 6. Comparison of our algorithm with a laser technique.

\section{Conclusions}

This study presented the "natural" magnetization in the automobile wheels. This magnetization is due to the history of the steel belt. Each tire has a different magnetization but this magnetization remains the same for every revolution of the wheel.

In this study we have presented a simple mechanical model of the wheel and the suspension. With this model we made a link between the wheel clearance we want to estimate and the magnetic signals we get from two sensors located on the chassis.

We have compared this technique with a laser sensor that measures the global clearance of the car as a reference and we get good results. In the future it would be interesting to test the system with an exact wheel clearance reference sensor.

This article showed the interest of using the magnetization of steel parts of a vehicle. With the development of high sensitivity sensors it is now possible to imagine magnetic applications for measuring displacements without putting a magnet on the part we want to monitor.

\section{References}

[1] P. Loane Edward, Speed and depth effects in magnetic anomaly detection advanced naval vehicle concept evaluation study, Office of the Chief of Naval Operations (OP. 96 V), 1976.

[2] G. Nichols, Rotational position and velocity sensing apparatus, US Patent No 4373486, 1983.

[3] K. Masahiro, Method and apparatus for detecting tire revolution using magnetic field, US Patent No 6246226, 2001.

[4] A. Le Goff, J. Lacoume, R. Blapain, in: COMADEM. Advances in maintenance and condition diagnosis technologies towards sustainable society, 2010.

[5] J. Brossard, Dynamique du véhicule, Modélisation des systémes complexes, Presses polytechniques et universitaires romandes, 2006.

[6] N. Melinat, Method and Device for the Determination of the Road Clearance of a Vehicle, US Patent No 309325, 2008.

[7] F. Bonnardot, Comparaison entre les analyses angulaire et temporelle des signaux vibratoires de machines tournantes. Étude du concept de cyclostationnarité floue, Ph.D. Thesis, INPG, 2004.

[8] M. Unser, IEEE Signal Processing Magazine 16 (1999) 22-38.

[9] V. Cattin, S. Dauvé, ATZ-Autotechnology 8 (2008) 56-59. 